\title{
Inclinometric method for determining the mechanical state of an overhead power transmission line
}

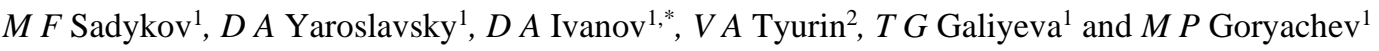 \\ ${ }^{1}$ Institute of Electric Power Engineering and Electronics, Kazan State Power Engineering University, Kazan, Krasnoselskaya str., 51 , \\ 420066, Russian Federation \\ ${ }^{2}$ Kazan (Volga region) Federal University, Kazan, Kremlin str., 18, 420008 ,Russian Federation
}

\begin{abstract}
Mechanical deformations when a wire is stretched causes its rotation around its axis, which allows obtaining additional information about the wire behavior in an overhead transmission line and considering it no longer as a group of sections from one span but as a whole interconnected section with redistribution of mechanical loads between spans. To determine the tensile strength of wire by its torsion, a method for determining the mechanical parameters of an overhead transmission line was developed. It is based on the inclinometric method and the theory of force calculation of steel ropes. This technique takes into account the torsion, the angle of inclination and the wire temperature. The technique is implemented in the system for monitoring the status of overhead power lines. It takes into account the torsion angle of the wire and helps to prevent emergencies on the overhead power line by determining the tensile strength of the wire, checking for defects in wires of the overhead power line and defects in the suspension armature. The monitoring system includes control devices, data collection and data processing center, a dispatcher software package. Control devices are installed directly on the wire/ground wire of an overhead power line and are used to measure the angle of rotation, the angle of inclination and the wire temperature. The data collection and data processing station processes them according to the developed methodology in specialized software. The system for monitoring the status of overhead power lines based on the inclinometric method helps to prevent emergencies and reduce the economic costs of maintaining and restoring overhead power lines.
\end{abstract}

\section{Introduction}

Transmission of electrical energy from power plants or substations to the consumer is carried out by power lines (mainly air) which are part of an electrical system. An overhead power transmission line (OHL) is a device for transmitting electrical energy through wires located in the open air and secured with insulators and line fittings to the supports.

The length of overhead transmission lines in the Russian Federation is over 2.8 million $\mathrm{km}$ [1]. Grid economy is rapidly becoming obsolete. The level of equipment wear reaches $70 \%$. At the same time, due to growth of electricity consumption and commissioning of new sections of OHL, the need for monitoring and diagnostics the $\mathrm{OHL}$ state and predicting possible emergencies increases [2].

The integral elements of an overhead transmission line are wire, insulators with linear armature and supporting structures (supports and foundations). These elements experience mechanical loads and may be damaged if they exceed their mechanical strength limit [3]. Overhead power lines are constructed in areas with various climatic conditions that affect the mechanical strength of the OHL elements and the nature of external influences.

Constructive elements of an overhead transmission line are tested using both horizontal (for example, mechanical stresses in the tensioned wire) and vertical loads (for example, from their own weight or from the weight of the wire). The wire is an element of an overhead line for which changes in mechanical loads are observed. This is caused by the change in the tension force of the wire depending on the temperature, the formation of icy-frost deposits on it, and fluctuations due to wind gusts. The main mechanical stresses in the overhead transmission line wires are related to its stretching. With an increase in the tensile strength of wire or core, from which it is made, the tension, that it is able to withstand, increases $[4,5]$.

Mechanical overload of the structural element of the overhead transmission line may occur due to appearance of icy-frost deposits, development of fatigue of the structural element of the overhead line, errors in line construction, repair and restoration work. Among these reasons, the most serious consequences are accompanied by the appearance of icy-frost deposits. Thus, a sufficiently precise localization of the emergency site or defect is necessary, provided that the passage along the

* Corresponding author: divanale@gmail.com 
overhead transmission line is often difficult (deep snow drifts; natural obstacles, marshland, terrain, etc.), and the speed of preventing/eliminating an emergency situation is directly related to incurred losses.

\section{Model of Inclinometric Method}

A promising method for determining the mechanical loads on a wire is the inclinometric method, based on measuring the angle of inclination of various objects relative to the gravitational field of the earth. The accuracy of this method can be improved by installing sensors with high accuracy of determining the angle of inclination of the wire, using the method of converting data from the device into the current mechanical loads on the wire [6, 7]. This is possible as when a wire/ground wire is stretched, its rotation around its axis is observed, which allows obtaining additional information and, therefore, to improve the inclinometric method.

Wires and grounding wires for overhead lines have a multi-wire structure and are a rope twisted from separate wires and possessing great flexibility.

After twisting, each wire, except for one central wire, is located along a helix. Fig. 1 shows images of wires and ground wires used at overhead lines.

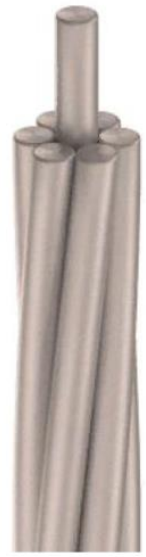

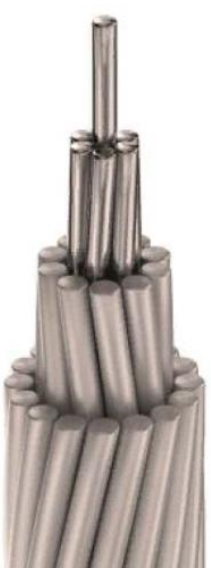

b

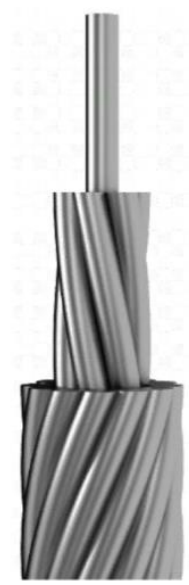

c
Fig. 1. Wires and ground wires: a - A-70; b - AC - 120/19; c ground wire SCC -50 .

Mechanical deformations allow observing a wire/ground wire rotation around its axis when it is stretched [7-10], to obtain additional information and to view overhead lines not as a group of sections from one span, but as a whole interconnected section with redistribution of mechanical loads between spans.

Based on the fact that multiwire wires and ground wires are similar in structure to the ropes, to evaluate their rotation around their axis, we use the theory of force calculation of steel ropes [3], which takes into account the temperature expansion and elastic deformation in the wire as compared to the existing inclinometric method. Thus, the dependence of the tension force of the wire on the torsion angles and the slope and temperature of the wire was obtained.

$$
\begin{aligned}
& T=\left(\alpha^{*}\left(l+L_{0} \cdot \operatorname{ch}(u)\right)-\right. \\
& \left.-\sqrt{\left(\alpha^{*}\right)^{2}\left(l+L_{0} \cdot \operatorname{ch}(u)\right)^{2}-8\left(\alpha^{*}\right)^{2} L_{0}\left(\varphi_{1}-\varphi_{0}\right) \frac{B}{C}}\right) / \\
& / 2 \alpha^{*} L_{0} \cdot \cos (\alpha)
\end{aligned}
$$

where $\alpha^{*}$ is the specific elongation of the wire, $\mathrm{N}$;

$l$ is the span length, $\mathrm{m}$;

$u=\ln \operatorname{tg}\left(\frac{\pi}{4}+\frac{\alpha}{2}\right)$,

$\alpha$ is the angle of inclination of the wire at the suspension point with respect to the straight line passing through the suspension points of this span;

$B$ is the wire stiffness coefficient, $\mathrm{N} \cdot \mathrm{m}^{2}$;

$\mathrm{C}$ is the wire stiffness coefficient, $\mathrm{N} \cdot \mathrm{m}$;

$L_{0 \mathrm{~g}}$ is the length of the unstretched wire at the current temperature, $\mathrm{m}$;

$\varphi_{1}$ is the angle of rotation of the wire around its axis at the current mechanical loads on the wire at ambient temperature until the appearance of icy-frost deposits, degrees;

$\varphi_{0}$ is the initial angle of rotation of the wire around its axis until the appearance of the icy-frost deposits, degrees.

Numerical calculation of the parameters of the ground wire in the span shows that the model reacts to changes in the torsion angle, confirming the correctness of the physical and mechanical principles embodied in it.

However, in practice, it is possible to use this technique based on the improved inclinometric method only at torsion angles of a wire/ground wire up to $180^{\circ}$, which is explained by the design limitations of the wire/ground wire torsion when fastening it to a support. Thus, the diameter of the icing clutch when calculating the C-50 thunderstorm should not exceed $12.5 \mathrm{~mm}$ and it is correct only for spans with one anchor support.

The resulting dependence of the torsion angle of the ground wire on its tension force in the range of practical application of the developed technique is presented in Fig.2.

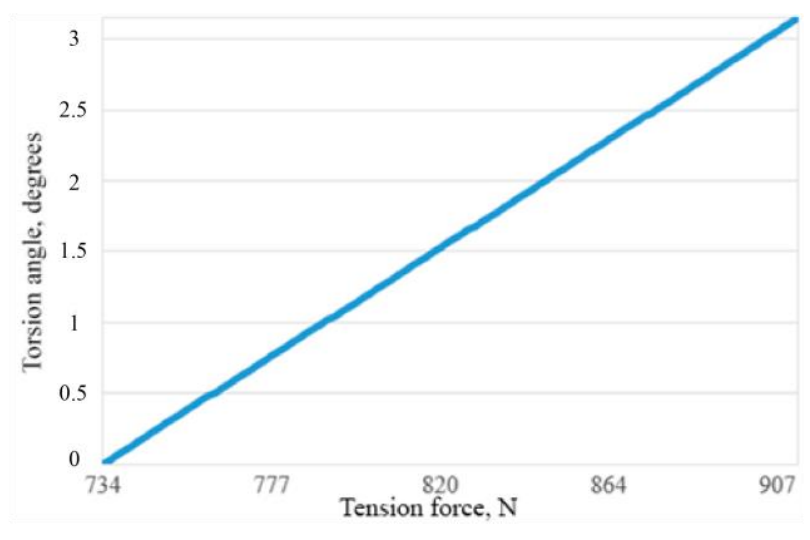

Fig. 2. The dependence of the torsion angle of the ground wire on its tension force in the range of practical application of the developed technique.

The advantages of the inclinometric method model are:

- The ability to assess the elastic interactions in the wire/ground wire; 
- The possibility of assessing the temperature impact on the mechanical loads of the wires/ground wires;

- The dependence of the parameters of mechanical loads on the torsion angle of the wire/ground wire around its axis, which is important in view of the greater range of operation of the control device on the torsion angle than on the angle of inclination;

- The ability to determine the initial torsion angle of the control device due to the calibration stage.

\section{Hardware and software}

The system for monitoring the status of overhead power lines consists of a special set of hardware-metrological tools (control devices) and specialized software. The control devices [11] are fixed on the wire/ground wire near the suspension point. Data from control devices is collected at the data collection point, and then transferred to the cloud server then they arrive at PC, where they are processed according to the developed techniques using the specialized software. The processed data is available for dispatching and monitoring via the web interface.

The software works with data on both the angle of inclination and on torsion angle of the wire when determining mechanical loads on overhead lines [12]. The mathematical model of the software calculates the strength of the wire based on the values of physicomechanical characteristics of the wires, the length of the unstretched wire, and the parameters measured by the monitoring devices $[13,14]$.

\section{Practical application of the developed system for monitoring the status of overhead power lines}

The system for monitoring the status of overhead power lines determines the mechanical loads on the wires/ground wires of overhead lines. Mainly, mechanical loads are caused by temperature changes in the wire/ground wire geometry, the appearance of icyfrost deposits on overhead lines/ground wires, as the ends of the wire/ground wire in the overhead line are rigidly fixed with fasteners. Therefore, the determination of the mechanical parameters of the OHL allows inspection of both icy-frost deposits and defects on the overhead power lines.

To verify the developed method, a comparison was made with the classical inclinometric method using the obtained tension at the suspension point. The initial data for the calculation are the real values of the angles of sag and torsion of the wire obtained from the monitoring devices of the OHL monitoring system.

The calculation of the tension force for a $6 \mathrm{kV}$ overhead line with an A-70 wire was made based on real data for cases with and without icy-frost deposits.

The calculation of overhead lines without icy-frost deposits was carried out for a span of $40.9 \mathrm{~m}$ length with walk-through supports. The geometrical parameters of the OHL were determined on the basis of photogrammetry of the obtained images of the OHL $[7$, $15,16]$. The temperature of the wire varied from -7.40 ${ }^{\circ} \mathrm{C}$ to $13.05{ }^{\circ} \mathrm{C}$. The angle of inclination of the wire changed from $1.76^{\circ}$ to $3.10^{\circ}$ and the torsion angle of the wire changed from $1.75^{\circ}$ to $4.14^{\circ}$ during this time. The calculation was carried out according to the angle of inclination of the wire and the angle of its torsion. The resulting force of tension according to the old method varies from $942 \mathrm{~N}$ to $1595 \mathrm{~N}$, and according to the new method from $942 \mathrm{~N}$ to $1649 \mathrm{~N}$.

The diagram describing the case without icy-frost deposits is shown in Fig.3.

From the diagram in Fig. 3 it follows that the wire tension forces calculated on the basis of the classical method and the newly developed one coincide. This proves the efficiency of the developed model. From the diagram it can be seen that with an increase in temperature, the angle of inclination increases and the wire unwinds. At the same time, the tension force of wire decreases. The behavior of the developed model does not contradict the physical laws of thermal expansion of bodies.

The calculation of the overhead line with icy-frost deposits was carried out for a span of $39.6 \mathrm{~m}$ length with one anchor support. The geometrical parameters of OHL were determined on the basis of photogrammetry of the obtained images [7, 15, 16].

The diagram describing the case of icy-frost deposits is shown in Figure 4.

The wire temperature varied from $-6.93{ }^{\circ} \mathrm{C}$ to 0.29 ${ }^{\circ} \mathrm{C}$. The angle of wire changed from $2.22^{\circ}$ to $3.8^{\circ}$ and the torsion angle of wire changed from $15.64^{\circ}$ to $35.54^{\circ}$ during this time. The calculation was carried out according to the classical and a new method. The resulting force of tension calculated according to the classical method varies from $1162 \mathrm{~N}$ to $2252 \mathrm{~N}$ and from $1161 \mathrm{~N}$ to $2254 \mathrm{~N}$ when calculated using the new one.

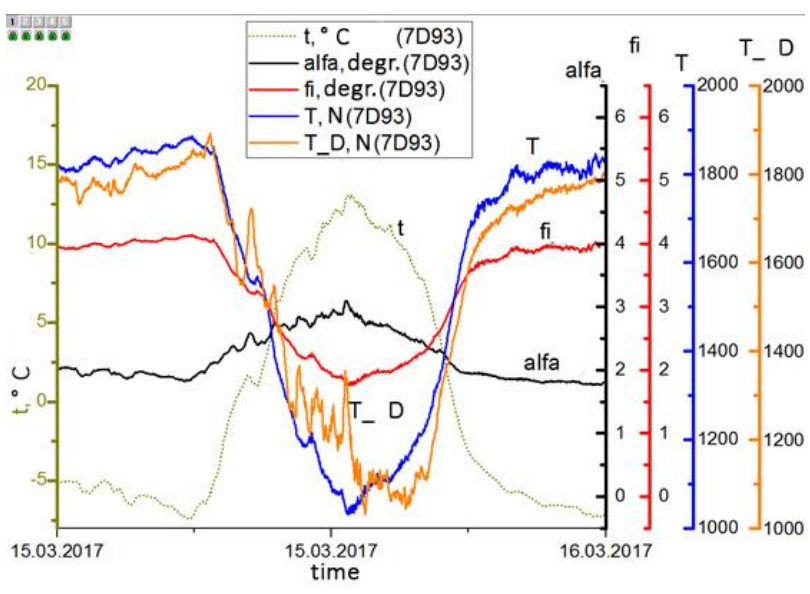

Fig. 3. Wire behavior in the absence of the icy-frost deposits: $t$ is the wire temperature; alfa is the wire angle; $f i$ is the wire torsion angle; $T g D$ is the wire force, calculated using the old method; $T \_g$ is the wire force, calculated using the new method.

By comparing these two cases, it can be seen that there is an increase in the ratio of change range in the rotation angle with respect to the change range in the 
angle of inclination of the wire with an increase in the force of tension acting on the wire.

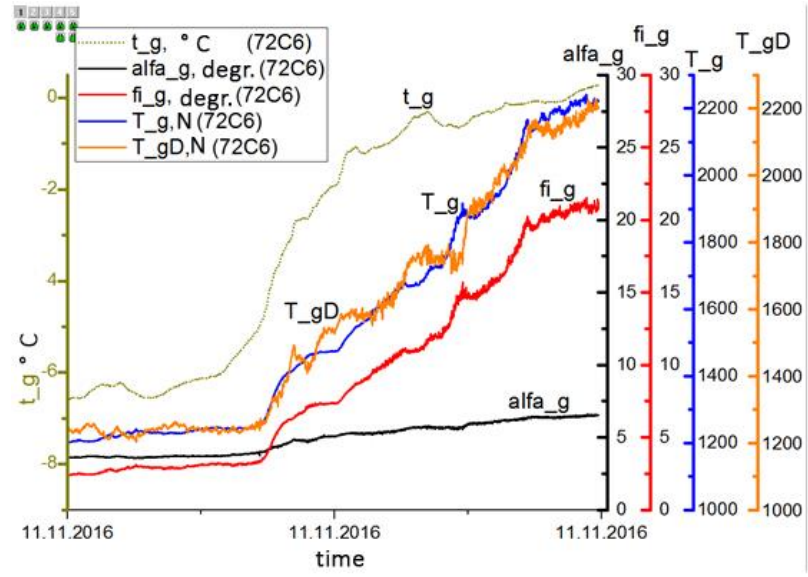

Fig. 6. Wire behavior with icy-frost deposits: $t$ is the wire temperature; alfa is the angle of inclination of the wire, $f i$ is the torsion angle of the wire; $T \_g D$ is the force of the wire, calculated using the old method; $T \_g$ is the force of the wire, calculated using the new method.

\section{Conclusion}

The practical application of the system for monitoring the status of overhead power lines on the basis of an inclinometric method is substantiated by determining mechanical loads on overhead lines/ground wires both in the presence and in the absence of icy-frost deposits.

\section{Acknowledgements}

The reported study was funded by RFBR according to the research project № 18-08-00203.

\section{References}

1. G. Bokov, Technical re-equipment of Russian electrical networks. How much can it cost?, Electric news, 2(14) (2002) URL: http://www.news.elteh.ru/arh/2002/14/03.php.

2. E.I. Satsuk, Software and hardware for monitoring overhead power lines and managing the power system in extreme weather conditions: PhD Thesis, 314 (2011)

3. A.D. Boshnyakovich, Mechanical calculation of wires and cables of power lines, 254 (1962)

4. D. Merkin, Introduction to the mechanics of a flexible thread, 40 (1980)

5. L. Keselman, Basics of mechanics of overhead power lines, 352 (1992)

6. M.V. Panasenko, Analytical review of methods and devices for monitoring the intermediate span of an overhead power line, International Journal of Applied and Fundamental Research, 11(4), 572-576 (2014)

7. D.A. Yaroslavsky, M.F. Sadykov, D.A. Ivanov, M.P. Goryachev, A.B. Konov, Methodology of ice coating monitoring on overhead transmission lines considering misalignment using wireless communication channel sensors, ARPN Journal of Engineering and Applied Sciences, 12(22), 64796482 (2017)

8. T. Otto, Integrated Microsystems for Smart Applications, Sensors and Materials, 30(4), $767-$ 778 (2018)

9. D.A. Ivanov, O.G. Savelyev, R.S. Misbakhov, System of monitoring and quantitative control of ice formation on wires of air transmission lines. Energy, electromechanics and energy-efficient technologies through the eyes of youth, the materials of the IV Russian Youth Scientific School-Conference, 334-336 (2016)

10. M. Muhr, S. Pack, S. Jaufer, Elektrotech. Inftech, 444 (2008) https://doi.org/10.1007/s00502-0080597-x

11. M.F. Sadykov, M.P. Goryachev, D.A. Ivanov, D.A. Yaroslavsky, I.M. Koryshkin, The patent of the Russian Federation №185311. Device for operational monitoring of the technical condition of high-voltage power lines, 2018120028 (2018)

12. D.A. Yaroslavskiy, M.F. Sadykov, Development of sensor system for monitoring and control of quantitative freezing for overhead lines power, Power engineering: research, equipment, technology, $\quad \mathbf{1 9 ( 3 - 4 ) ,} \quad 69-79 \quad$ (2017) https://doi.org/10.30724/1998-9903-2017-19-3-469-79

13. M.P. Goryachev, D.A. Yaroslavsky, A.A. Abdullov, Z.G. Baymukhametov, Analysis of the geometric parameters of power lines for calibration of sagging wires, Proceedings of the XII All-Russian Open Youth Scientific-Practical Conference "Dispatching and Control in the Electric Power Industry", 470 -475 (2017)

14. D.A. Yaroslavskiy, M.F. Sadykov,M.P. Goryachev, D.A. Ivanov, T.G. Yambaeva, Investigations of topological features of construction an intelligent overhead power transmission line based on wireless sensors, International Journal of Mechanical Engineering and Technology, 8(12), 903-908 (2017)

15. D. Efanov, D. Sedykh, G. Osadchy, D. Barch, Permanent monitoring of railway overhead catenary poles inclination, IEEE East-West Design \& Test Symposium (EWDTS), 1-5 (2017) doi: 10.1109/EWDTS.2017.8110142

16. $\mathrm{Y} . \mathrm{Hu}, \mathrm{K}$. Liu, Inspection and Monitoring Technologies of Transmission Lines with Remote Sensing, ISBN 9780128126448, 281-508 (2017) https://doi.org/10.1016/B978-0-12-8126448.00005-9. 